\title{
ON A QUESTION CONCERNING COUNTABLY GENERATED $z$-IDEALS OF $C(X)$
}

\author{
ATTILIO LE DONNE
}

\begin{abstract}
In $\left[D_{1}\right]$ the following question was asked: is every countably generated $z$-ideal of $C(X)$ of the form $O^{A}=\bigcap_{p \in A} O^{p}$, for some zero-set $A$ of $\beta X$ ? It is proved here that the answer is affirmative when $X$ is normal and first countable; and an example is given, disproving the general conjecture. For terminology and notation see [GJ], [ $\left.\mathbf{D}_{\mathbf{1}}\right]$.
\end{abstract}

\section{Countably generated $z$-ideals.}

1.1. First of all, we observe that the question asked in $\left[D_{1}\right]$ may be reformulated in a purely algebraic way: that is, it involves $C(X)$ only, and not the underlying space $X$, which need not even be assumed completely regular.

Their usual definition notwithstanding, $z$-ideals are purely algebraic objects: see [GJ, 4A.5]. And ideals of the form $O^{A}=\bigcap_{p \in A} O^{p}$, with $A$ being a closed subset of $\beta X$, may also be defined algebraically, with no reference to $X$ or $\beta X$ : it is proved in $[\mathrm{Br}]$, in $[\mathrm{Bk}]$ or in $\left[\mathrm{D}_{2}\right]$ that such ideals are exactly the pure ideals of $C(X)$ (we recall that an ideal $I$ of a commutative ring $R$ (with 1 ) is said to be pure if for every $f \in I$ there exists $g \in I$ such that $f=f g$ ). In [ $\mathbf{D}_{1}$, Lemma 2.1], it is proved that $O^{A}$ (with $A$ closed in $\beta X$ ) is countably generated if and only if $A$ is a zero-set of $\beta X$.

Thus the question under investigation may be restated:

Is every countably generated $z$-ideal of $C(X)$ a pure ideal?

1.2. As customary, given a subset $\left\{f_{\alpha}\right\}$ of $C(X),\left(f_{\alpha}\right)$ denotes the ideal generated by that subset; in particular, if $g \in C(X)$, then $(g)$ denotes the principal ideal generated by $g$.

If $f, g \in C(X)$ and $Z(f)$ is a neighborhood of $Z(g)$ in $C(X)$ then $f$ is a multiple of $g$ in $C(X)$ [GJ, 1.0]. Hence, with $\omega=$ the natural numbers, we have the following:

LEMMA. Let $\left(f_{n}\right)_{n \in \omega}$ be a sequence in $C(X)$ such that int ${ }_{X}\left(Z\left(f_{n}\right)\right) \supseteq Z\left(f_{n+1}\right)$, for every $n \in \omega$. Then $I=\left(f_{0}, f_{1}, f_{2}, \ldots\right)$ is a $z$-ideal.

1.3 LemMa. Let I be an ideal of $C(X)$. Then

(i) $I$ is pure if and only if for each $f \in I$ there exists $g \in I$ such that $X \backslash Z(f)$ and $Z(g)$ are completely separated in $X$.

(ii) If $X$ is normal, then $I$ is pure if and only if for every $f \in I$ there exists $g \in I$ such that int $_{X} Z(f) \supseteq Z(g)$,

Received by the editors August 1, 1977 and, in revised form, July 25, 1979.

AMS (MOS) subject classifications (1970). Primary 54B17, 54D35; Secondary 54D60. 
Proof. (i) By definition of purity (cf. 1.1) $I$ is pure if and only if given $f \in I$ there exists $g \in I$ such that $f=f g$. If $I$ is pure, then $1-g$ completely separates $X \backslash Z(f)$ and $Z(g)$; conversely, if $f, g \in I$, and $v \in C(X)$ is 1 on $X \backslash Z(f)$ and 0 on a neighborhood of $Z(g)$, then $f=f v$, and $v \in I$, being a multiple of $g$. Thus $I$ is pure.

(ii) follows obviously from (i) since, in normal spaces, two subsets are completely separated iff they have disjoint closures.

1.4 LEMMA. Every countably generated semiprime ideal I of $C(X)$ is generated by a sequence $g_{0}, g_{1}, g_{2}, \ldots$ such that

$$
\left(g_{0}\right) \subset\left(g_{1}\right) \subset\left(g_{2}\right) \subset \cdots .
$$

Proof. If $I=\left(f_{0}, f_{1}, f_{2}, \ldots\right)$, put $g_{n}=\sum_{i=0}^{n}\left|f_{i}\right|^{1 / 2}$ (cf. [D, 3]).

When $X$ is first countable, there is a converse to Lemma 1.2:

1.5 LEMMA. Assume $X$ is first countable. Then every countably generated $z$-ideal $I$ of $C(X)$ has a sequence of generators $f_{0}, f_{1}, f_{2}, \ldots$ such that int $X_{X} Z\left(f_{n}\right) \supset Z\left(f_{n+1}\right)$, for every $n \in \mathbf{N}$.

Proof. Being a $z$-ideal, $I$ is semiprime, hence it has a sequence of generators $g_{0}$, $g_{1}, g_{2}, \ldots$ with $\left(g_{0}\right) \subset\left(g_{1}\right) \subset\left(g_{2}\right) \subset \cdots$ by Lemma 1.4. We prove that $g_{0}, g_{1}$, $g_{2}, \ldots$ admits a subsequence with the required property. Put $n(0)=0$. If int $_{X} Z\left(g_{0}\right)$ fails to contain any $Z\left(g_{n}\right)$, then for each $n$ there exists $p_{n} \in \operatorname{cl}_{X} \operatorname{coz}\left(g_{0}\right)$ $\cap Z\left(g_{n}\right)$ and a sequence $\left(x_{m}^{n}\right)_{m}$ in $\operatorname{coz}\left(g_{0}\right)$, which converges to $p_{n}$ as $m \rightarrow \infty$. For each $n$, the set $S_{n}=\operatorname{cl}_{\beta X} Z\left(g_{0}\right) \cup\left\{x_{m}^{n}: m \in N\right\}$ is then a closed subset of $\beta X$; the function which is zero on $\operatorname{cl}_{\beta X} Z\left(g_{0}\right)$ and $\left|g_{n}\left(x_{m}^{n}\right)\right|^{1 / 2}$ on $\left\{x_{m}^{n}: m \in N\right\}$ is clearly continuous on $S_{n}$; hence it has a continuous extension $h_{n}^{*} \in C(\beta X)$. Let $h_{n}=$ $h_{n}^{*} \mid X$. Put $h=\sum_{n=0}^{\infty}\left(h_{n} \wedge 2^{-n}\right)$; by uniform convergence, $h \in C(X)$. Clearly $Z(h)$ $\supset Z\left(g_{0}\right)$; but $h$ cannot be a multiple (in $C(X)$ ) of any $g_{n}$. For if $h=u g_{k}$, then $u g_{k}>h_{k} \wedge 2^{-k}$; if $m$ is sufficiently large, we have $h_{k}\left(x_{m}^{k}\right) \wedge 2^{-k}=\left|g_{k}\left(x_{m}^{k}\right)\right|^{1 / 2}$, hence $\left|u\left(x_{m}^{k}\right)\right|>2^{-k}\left|g_{k}\left(x_{m}^{k}\right)\right|^{-1 / 2}$; letting $m \rightarrow \infty$, we see that $u$ cannot be continuous at $p_{k}$. This contradiction shows that there exists $n(1) \in N$ such that int ${ }_{X} Z\left(g_{0}\right)$ $\supset Z\left(g_{n(1)}\right)$. An obvious induction yields the desired subsequence.

1.6 Proposition. If $X$ is normal and first countable, then every countably generated $z$-ideal of $C(X)$ is pure. Hence, in such spaces, countably generated $z$-ideals are the ideals $O^{A}$, with $A$ running on the zero-sets of $\beta X$.

Proof. By Lemmas 1.5 and 1.3(ii).

2. The example. In this section we describe a space $X$ and a countably generated $z$-ideal $I$ of $C(X)$ that is not pure. (What we construct is a space $X$ and a sequence $\left(Z_{n}\right)_{n \in \omega}$ of zero-sets of $X$ such that int $X_{n} \supset Z_{n+1}$, but $X \backslash Z_{0}$ and $Z_{n}$ are not completely separated, for every $n \in \omega$; this disproves the conjecture in question (Lemma 3(a) and Lemma 2, \$1).)

The space is obtained by attaching together, in a certain way, infinitely many copies of Tychonoff planks; $\omega=\omega_{0}$ and $\omega_{1}$ are the cardinals $\aleph_{0}, \kappa_{1}$, respectively, 
considered as initial ordinals, with their order topology. Consider the (complete) Tychonoff planks $T^{*}=\left(\omega_{1}+1\right) \times(\omega+1)$; let $S^{*}$ denote the space obtained from $T^{*}$ by identifying all points of the "top edge" $\left(\omega_{1}+1\right) \times\{\omega\}$ to a point $c$. It is straightforward to see that $S^{*}$ is a compact Hausdorff space with clopen basis. (One can picture it as a set of "rays" centered at $c$, each ray being a copy of $\omega+1$, with $c$ as limit point.) The (complete) book is the space $B^{*}=S^{*} \times\left(\omega_{1}+1\right)$, with product topology. Clearly, $B^{*}$ is a compact Hausdorff space with clopen basis. The subspace $W^{*}=\{c\} \times\left(\omega_{1}+1\right)$ is a copy of $\omega_{1}+1$; it is called the back of $B^{*}$. Points of $B^{*} \backslash W^{*}$ are triples $(\alpha, m, \beta)$ with $\alpha, \beta \in \omega_{1}+1, m \in \omega ; \alpha, m, \beta$ may be thought of as "cylindrical coordinates" $\alpha$ being the angle, $m$ the radius, $\beta$ the height; but it is more fitting to think of $\alpha$ as the page in which the point lies, and $m$, $\beta$ as the column and the line, respectively at which one finds the point on the $\alpha$ th page. Thus, the book has $\kappa_{1}$ pages, each page has $\omega=\kappa_{0}$ columns and $\kappa_{1}$ lines. For a better understanding of the arguments which follow it is useful to keep this picture in mind. The top section of $B^{*}$ is the subspace $S^{*} \times\left\{\omega_{1}\right\}$; for each $m \in \omega$ we have the $m$ th top column $C_{m}=\left\{\left(\alpha, m, \omega_{1}\right): \alpha \in \omega_{1}+1\right\}$ it is a copy of $\omega_{1}+1$. The vertex of $B^{*}$ is the point $v=\left(c, \omega_{1}\right)\left(\in W^{*}\right)$. The top edge $E$ of $B^{*}$ is the subspace $\left\{\left(\omega_{1}, m, \omega_{1}\right): m \in \omega\right\} \cup\{v\}$; it is a copy of $\omega+1$. The incomplete book $B$ is the subspace $B^{*} \backslash E$.

We need some facts on $B, B^{*}$.

2.1 LemMA. (i) $W^{*}$ is a zero-set of $B^{*}$.

(ii) $B$ is an open dense $C$-embedded subspace of $B^{*}$. (Hence, $B$ is pseudocompact, and $B^{*}=\beta B$.)

Proof. (i) Define $\phi: B^{*} \rightarrow \mathbf{R}$ to be 0 on $W^{*}$, and put $\phi(\alpha, m, \beta)=2^{-m}$ for every $(\alpha, m, \beta) \in B^{*} \backslash W^{*}$. It is easy to see that $\phi$ is continuous. The proof of (ii) is deferred until $\$ 3$.

Consider now the space $\Lambda^{*}=(\omega \times \omega) \times B^{*}$, with product topology. Since $\omega \times \omega$ is discrete, $\Lambda^{*}$ is simply a topological sum of $\aleph_{0}$ disjoint copies of $B^{*}$, it is locally compact and $\sigma$-compact (hence realcompact) but not compact. It is called the (complete) library; its subspace $\Lambda=(\omega \times \omega) \times B$, the incomplete library, is open dense and $C$-embedded in $\Lambda^{*}$ (Lemma 2.1(ii)); then $\Lambda^{*}$ is the realcompactification of $\Lambda$. Given $r \in \omega$, the subspace $\Sigma_{r}^{*}=(\{r\} \times \omega) \times B^{*}$ is called the (complete) $r$ th shelf of $\Lambda^{*}$; and $(\{r\} \times\{s\}) \times B^{*}$ is the sth book of the $r$ th shelf (the meaning of $\Sigma_{r}$, incomplete $r$ th shelf, should be obvious). We now "attach" to each other the books of $\Lambda^{*}$ in a certain way; we shall obtain a quotient $X^{*}$ of $\Lambda^{*}$, which will have the image $X$ of $\Lambda$ as dense and $C$-embedded subspace; the space $X$ will yield our example. Fix a bijection $u: \omega \rightarrow \omega \times \omega, u(s)=\left(s_{1}, s_{2}\right)$. Define a (noncontinuous) map $\mu: \Lambda^{*} \rightarrow \Lambda^{*}$ piecewise, as follows: points of $\Lambda^{*}$ which do not belong to the back of any book are left fixed; if $p$ lies in the back of the sth book of the $r$ th shelf, say $p=((r, s),(c, \beta)), \quad \beta \in \omega_{1}+1$, then define $u(p)=\left(\left(r+1, s_{1}\right)\right.$, $\left.\left(\beta, s_{2}, \omega_{1}\right)\right)$. In other words, $\mu$ "attaches" the back of the $s$ th book of the $r$ th shelf (this back is a copy of $\omega_{1}+1$ ) to the $s_{2}$ th top column of the $s_{1}$ th book of the $(r+1)$ st shelf. (This column is a copy of $\omega_{1}+1$, too.) The equivalence relation 
identifies $x$ and $\mu(x)$, for every $x \in \Lambda^{*}$; since $\mu$ is idempotent (i.e. $\mu \circ \mu=\mu$, as is easy to see) each equivalence class contains at most two points (exactly one if the point does not lie on the back or on the "top section" $S^{*} \times\left\{\omega_{1}\right\}$ of any book); this implies that the quotient is a $T_{1}$-space (but we do not need, at this point, any separation property for the quotient; in $\$ 3$ it shall be proved that the quotient is a $T_{4}$ zero-dimensional space).

Let $X^{*}$ be the quotient, $q: \Lambda^{*} \rightarrow X^{*}$ the quotient map. It is easy to verify that $q^{\leftarrow}(q(\Lambda))=\Lambda$ (vertices of books are mapped by $\mu$ into points of top edges); since $\Lambda$ is open (and dense) in $\Lambda^{*}$, this implies that $X=q(\Lambda)$ is open (and dense) in $X^{*}$, moreover the quotient topology of $X$ (via the map $q \mid \Lambda$ ) coincides with the subspace topology.

By well-known properties of quotients, $f \in C\left(\Lambda^{*}\right)$ may be factored as $g \circ q$, with $g \in C\left(X^{*}\right)$ if and only if $f$ is constant on the equivalence classes of $q$; this may be restated as $f \circ \mu=f$. The same holds true for $\Lambda$ and $X$, of course. We claim that

2.2 Lemma. $X$ is dense and is $C$-embedded in $X^{*}$. Hence $C(X)$ and $C\left(X^{*}\right)$ are isomorphic.

Proof. Given $g \in C(X)$, consider $h=g \circ(q \mid \Lambda) \in C(\Lambda)$. As observed before, $\Lambda$ is dense and $C$-embedded in $\Lambda^{*}$; let $h^{*}$ denote the continuous extension of $h$ on $\Lambda^{*}$. Since $h \circ \mu=h$, also $h^{*} \circ \mu=h^{*}$ (given a vertex of some book in $\Sigma_{r}^{*}, \mu$ maps it into some point of the top edge of some book of $\Sigma_{r+1}^{*}, h$ must eventually assume the same value on tails of the back of the book in the $r$ th shelf and in the top column of the book of the $(r+1)$ st shelf to which $\mu$ attaches this back). Hence $h^{*}$ factors as $g^{*} \circ q$, with $g^{*} \in C\left(X^{*}\right)$ being the required extension of $g$.

We now define a sequence $\left(f_{n}\right)_{n \in \omega}$ of functions of $C\left(\Lambda^{*}\right)$, compatible with the equivalence relation; the sequence $\left(g_{n}^{*}\right)_{n \in \omega}$ of $C\left(X^{*}\right)$ (where $g_{n}^{*} \circ q=f_{n}$ for each $n \in \omega)$ will be shown to generate a $z$-ideal of $C\left(X^{*}\right)$ which is not pure. Define $f_{n}$ to be identically zero on $U_{r>n+1} \Sigma_{r}^{*}$. This forces us to put $f_{n}$ identically zero on all the backs of books of $\Sigma_{n}^{*}$; we define $f_{n}$ on the other points of books of the $n$th shelf by means of the function $\phi$ used in the proof of Lemma 1(i) of this section. On the $m$ th top columns of books in the $n$th shelf, $f_{n}$ has then constant value $2^{-m}$; use this constant value to define $f_{n}$ on books of the $(n-1)$ st shelf attached to these columns. Repeating this last procedure, it is easy to define $f_{n}$ (piecewise constant) on all books of all shelves of lower degree. It is clear that $f_{n}$ is $q$-compatible, i.e. $f_{n}=g_{n}^{*} \circ q$ with $g_{n}^{*} \in C\left(X^{*}\right)$. Put $g_{n}=g_{n}^{*} \mid X$.

Denote by $I$ the ideal generated by $\left(g_{n}\right)_{n \in \omega}$ in $C(X)$, by $I^{*}$ the (isomorphic) ideal in the (isomorphic) ring $C\left(X^{*}\right)$.

2.3 LEMMA. (i) $I$ is a $z$-ideal of $C(X)$.

(ii) $I^{*}$ is not pure in $C\left(X^{*}\right)$.

Proof (i) We prove that int $Z_{X} Z_{X}\left(g_{n}\right) \supseteq Z\left(g_{n+1}\right)$, for every $n \in \omega$ [cf. Lemma 1.3]. In fact $q^{\leftarrow}\left(Z_{X}\left(g_{n+1}\right)\right) \subseteq A \subseteq q^{\leftarrow}\left(Z_{X}\left(g_{n}\right)\right)$ where $A$ is $\cup_{r>n+1} \Sigma_{r}$ without the point of the "top sections" of the books of $\Sigma_{n+1}$, i.e. $A=\left(\cup_{r>n+1} \Sigma_{r}\right) \backslash q \leftarrow q \Sigma_{n}$. Since $A$ is an open subset of $\Lambda$ and is $A=q^{\leftarrow} q(A)$, the required result follows. 
(ii). We prove that $A=X^{*} \backslash Z_{X^{*}}\left(g_{0}^{*}\right)$ and $Z_{X^{*}}\left(g_{n}^{*}\right)$ are not completely separated, for any $n \in \omega$. In fact, the closure of $A$ in $X^{*}$ meets $Z_{X^{*}}\left(g_{n}^{*}\right)$, as we now show. Take any vertex of any book of the $r$ th shelf, for any $r>n$, say $((r, s), v)$. Then $p=q((r, s), v) \in Z_{X^{*}}\left(g_{n}^{*}\right)$; and any open neighbourhood $V^{*}$ of $p$ in $X^{*}$ meets $A$. For, $q^{\leftarrow}\left(V^{*}\right)$ is an open neighbourhood of $((r, s), v)$ in $\Lambda^{*}$; then $q^{\leftarrow}\left(V^{*}\right)$ contains infinitely many top columns of the sth book of the $r$ th shelf; by equivalence, $q^{\leftarrow}\left(V^{*}\right)$ contains also the backs of infinitely many books of the $(r-1)$ st shelf; it is then a neighbourhood of infinitely many vertices of books in this shelf, repeating the procedure, we see that $q^{\leftarrow}\left(V^{*}\right)$ is a neighbourhood of infinitely many vertices of books of $\Sigma_{0}^{*}$; thus $q^{\leftarrow}\left(V^{*}\right) \cap \operatorname{Coz}_{\Lambda^{*}}\left(f_{0}\right) \neq \varnothing$; hence $V^{*}$ meets $A=q\left(\operatorname{Coz}_{\Lambda^{*}}\left(f_{0}\right)\right)$.

3. In this section we give a proof of (ii) of Lemma 2.1 which asserts that $B$ is $C$-embedded in $B^{*}$.

We shall also prove that $X$ and $X^{*}$ are Hausdorff spaces with clopen basis.

Proof. Let $f \in C(B)$. We first prove that $f$ extends to a continuous function (which we still call $f$ ) on $B^{*} \backslash\{v\}$. Observe that for every $m \in \omega$ the subspace $C_{m}^{*}=\left\{(\alpha, m, \beta): \alpha, \beta \in \omega_{1}\right\}$ is clopen in $B^{*}$ and is homeomorphic to the space $\Omega^{*}=\left(\omega_{1}+1\right) \times\left(\omega_{1}+1\right)$ of [GJ, 8L]. From the same reference, we know that $\Omega=\Omega^{*} \backslash\left\{\left(\omega_{1}, \omega_{1}\right)\right\}$ is $C$-embedded in $\Omega^{*}$. Clearly $C_{m}=C_{m}^{*} \cap B$ is a copy of $\Omega$.

Given $f \in C\left(B^{*} \backslash\{v\}\right)$, observe that $f$ is eventually constant on the back, i.e. there exists $\bar{\beta} \in \omega_{1}$ such that $f(c, \beta)=f(c, \bar{\beta})$ for all $\beta \in \omega_{1}, \beta>\bar{\beta}$. For simplicity, assume this eventual value to be 0 ; and put $f(v)=0$. The extension so obtained is continuous: otherwise, there exist $\varepsilon>0$ and a sequence $p_{n}=\left(\alpha_{n}, m_{n}, \beta_{n}\right)$, with $\beta_{n}>\bar{\beta}, \beta_{n} \in \omega_{1}, \alpha_{n} \in \omega_{1}+1$, and $m_{n} \in \omega, \lim _{n \rightarrow \infty} m_{n}=\infty$, such that $\left|f\left(p_{n}\right)\right|>\varepsilon$. In the compact space $B^{*},\left(p_{n}\right)_{n}$ has cluster points; since $m_{n} \rightarrow \infty$ as $n \rightarrow \infty$, such a cluster point belongs to the back $W^{*}$; and since $\bar{\beta}<\sup _{n} \beta_{n}<\omega_{1}$, such a cluster point is necessarily some $\left(c, \beta^{\prime}\right)$, with $\bar{\beta}<\beta^{\prime}<\omega_{1}$. Then $f\left(c, \beta^{\prime}\right)=0$; but continuity of $f$ at $\left(c, \beta^{\prime}\right)$ implies $\left|f\left(c, \beta^{\prime}\right)\right|>\varepsilon$, a contradiction. This ends the proof.

Given a complete book $B^{*}$, and a point $(\bar{\alpha}, m, \bar{\beta}) \in B^{*} \backslash W^{*}$ a neighbourhood base for it in $B^{*}$ consists of "rectangles"

(1) $V_{\alpha, \beta}=\left\{(\xi, m, \eta) \in B^{*}: \alpha<\xi<\bar{\alpha}, \beta<\eta<\bar{\beta}\right\}$ with $\alpha<\bar{\alpha}, \beta<\bar{\beta}$.

For a point $(c, \bar{\beta}) \in W^{*}$ a neighbourhood base is

(2) $V_{m, \beta}=\{(c, \eta): \beta<\eta<\bar{\beta}\} \cup\left\{(\xi, n, \eta): \xi \in \omega_{1}+1, n>m ; \beta<\eta<\bar{\beta}\right\}$ with $m \in \omega, \beta<\bar{\beta}$.

Next, a neighbourhood base for the back $W^{*}$ of $B^{*}$ consists of the sets

(3) $V_{m}=W^{*} \cup\left\{(\alpha, n, \beta): \alpha, \beta \in \omega_{1}+1, n>m\right\}$, with $m \in \omega$.

All these sets are obviously clopen in $B^{*}$.

\subsection{Proposition. $X^{*}$ has a clopen basis.}

Proof. Take a point $p \in \Lambda^{*}, p$ belongs to some book of, say, the $n$th shelf $\Sigma_{n}^{*}$. If $p$ is neither in the back, nor in the top section (see \$2) of the book, then there exists a neighbourhood $V_{\alpha, \beta}$ of $p$ in the book (type 1) such that the equivalence relation is the identity on $V_{\alpha, \beta}$. Then $q^{\leftarrow}\left(q\left(V_{\alpha, \beta}\right)\right)=V_{\alpha, \beta}$, and $q(p)$ has a clopen neighbourhood basis in $X^{*}$. Assume now that $p=\mu\left(p^{\prime}\right)$, with $p^{\prime}$ in the back of some book in $\Sigma_{n-1}^{*}$. Take for $p^{\prime}$ a clopen neighbourhood $V_{1}$ of type $2, V_{m, \beta^{\prime}}$; take for $p$, in its 
book a neighbourhood $V_{0}$ (type 1) $V_{\beta^{\prime}, \beta}$. Assuming that $p^{\prime}$ is not the vertex of its book, we have $V_{0} \cup V_{1}=q^{\leftarrow} q\left(V_{0} \cup V_{1}\right)$, so that $q(p)$ has a clopen neighbourhood basis in $X^{*}$. Finally assume $p=\mu\left(p^{\prime}\right)$, with $p^{\prime}=v$, vertex of some book in $\Sigma_{n-1}^{*}$. Then the books of $\Sigma_{n-1}^{*}$ are either disjoint from $\mu^{\leftarrow}\left(V_{1}\right)$, or, otherwise, their back is contained in $\mu^{\leftarrow}\left(V_{1}\right)$. Choose a clopen neighbourhood of these backs of type 3 , and consider their union $V_{2}$. Repeating this procedure with $V_{2}$ in place of $V_{1}$, and so on inductively, we obtain a clopen subset of $\Lambda$ invariant under $\mu$ and $\mu^{\leftarrow}$; and the proof is complete.

3.2 Proposition. $X^{*}$ is a $\sigma$-compact Hausdorff space with clopen basis. Moreover, $X^{*}$ is the realcompactification of $X$.

Proof. As was observed in $\S 2, X^{*}$ is a $T_{1}$-space. Since $\Lambda^{*}$ is $\sigma$-compact, so is $X^{*}$. The remaining assertions follow easily.

ObSeRvation. It is not difficult to show that $X$ is locally compact (but not $\sigma$-compact, being not even realcompact). And $X^{*}$ is $\sigma$-compact, but not locally compact.

ADDED IN PROOF. The conjecture is true also for locally compact normal spaces. The proof, quite similar to the first countable case, will appear in a forthcoming paper.

\section{REFERENCES}

[Bk] Rudolphe Bkouche, Pureté, mollesse et paracompacité, C. R. Acad. Sci. Paris Ser. A 270 (1970), A1653-1655.

[Br] J. G. Brookshear, Projective ideals in rings of continuous functions, Pacific J. Math. 71 (1977), 313-333.

[D,] G. DeMarco, On the countably generated z-ideals of $C(X)$, Proc. Amer. Math. Soc. 31 (1972), 574-576.

$\left[\mathrm{D}_{2}\right] \ldots$, Projectivity of pure ideals (to appear).

[GJ] L. Gillman and M. Jerison, Rings of continuous functions, Van Nostrand, New York, 1960.

Seminario Matematico, University of Padova, Via Belzoni 7, 35100 Padova, Italy 